\title{
350-fold improved measurement of the antiproton magnetic moment using a multi-trap method
}

\author{
Christian Smorra ${ }^{1}$. Pascal E. Blessing ${ }^{1,2} \cdot$ Matthias J. Borchert ${ }^{1,3}$. Jack A. Devlin ${ }^{1}$. \\ James A. Harrington ${ }^{1,4}$. Takashi Higuchi ${ }^{1,5}$. Jonathan Morgner ${ }^{1,3}$. \\ Hiroki Nagahama ${ }^{1}$. Stefan Sellner ${ }^{1}$. Matthew A. Bohman ${ }^{1,4}$. Andreas H. Mooser ${ }^{1,4}$. \\ Georg L. Schneider ${ }^{1,6}$. Natalie Schön ${ }^{6} \cdot$ Markus Wiesinger ${ }^{1,4} \cdot$ Klaus Blaum $^{4}$. \\ Yasuyuki Matsuda ${ }^{5}$ Christian Ospelkaus ${ }^{3,7}$.Wolfgang Quint ${ }^{2}$.Jochen Walz $^{6,8}$. \\ Yasunori Yamazaki ${ }^{1}$. Stefan Ulmer ${ }^{1}$
}

Published online: 29 October 2018

(C) The Author(s) 2018

\begin{abstract}
We summarize our recent 1.5 parts per billion measurement of the antiproton magnetic moment using the multi Penning-trap system of the BASE collaboration. The result was achieved by combining the detection of individual spin-transitions of a single antiproton with a novel two-particle spectroscopy technique, which dramatically improved the data sampling rate. This measurement contributes to improve the test of the fundamental charge, parity, time reversal (CPT) invariance in the baryon sector by a factor of 350 compared to our last measurement, and by a factor of 3000 compared to the best competing measurement. We review the measurement technique and discuss the improved limits on CPT-violating physics imposed by this measurement.
\end{abstract}

Keywords Fundamental symmetries and interactions · CPT invariance $\cdot$ Standard model · Penning trap · Antiproton · Magnetic moments

This article is part of the Topical Collection on Proceedings of the 13th International Conference on Low Energy Antiproton Physics (LEAP 2018) Paris, France, 12-16 March 2018

Edited by Paul Indelicato, Dirk van der Werf, and Yves Sacquin

Christian Smorra

christian.smorra@ riken.jp

1 Ulmer Fundamental Symmetries Laboratory RIKEN, Wako, Japan

2 GSI-Helmholtzzentrum für Schwerionenforschung, Darmstadt, Germany

3 Institut für Quantenoptik, Leibniz Universität Hannover, Hannover, Germany

4 Max-Planck-Institut für Kernphysik, Heidelberg, Germany

5 Graduate School of Arts and Sciences, University of Tokyo, Tokyo, Japan

6 Institut für Physik, Johannes Gutenberg-Universität Mainz, Mainz, Germany

7 Physikalisch-Technische Bundesanstalt, Braunschweig, Germany

8 Helmholtz-Institut Mainz, Mainz, Germany 


\section{Introduction}

Antiprotons at ultra-low energy provide unique opportunities to directly test fundamental symmetries and interactions in the antimatter sector [1]. Precision spectroscopy on antiprotonic helium atoms [2] and antihydrogen [3-6] determine fundamental properties of antiparticles and their interactions, and provide stringent tests of CPT invariance. Other experiments aim to study the weak equivalence principle with antimatter [7-9].

Our experiments, BASE at CERN and the proton $g$-factor experiment BASE-Mainz, contribute to stringent tests of CPT invariance by comparing the fundamental properties of protons $[10,11]$ and antiprotons in Penning traps [12-15]. Other groups follow similar approaches [16, 17]. A single particle in a Penning trap forms a simple and well-understood system [18] and is therefore ideally suited to investigate and constrain CPT-odd physics [19-21]. In particular, the proton and antiproton charge-to-mass ratios $(q / m)_{p}$ and $(q / m)_{\bar{p}}$ and the magnetic moments, $\mu_{p}$ and $\mu_{\bar{p}}$, of the two particles can be compared by performing ultra-precise frequency ratio measurements [22]. The magnetic moment in units of the nuclear magneton $\mu_{N}$ is obtained by measuring the ratio of the antiproton's Larmor frequency (spin precession frequency) $v_{L}$ and its cyclotron frequency $v_{c}$ :

$$
\pm \frac{\mu_{p / \bar{p}}}{\mu_{N}}=\frac{g_{p / \bar{p}}}{2}=\left(\frac{v_{L}}{v_{c}}\right)_{p / \bar{p}},
$$

where $g_{p / \bar{p}}$ is the proton/antiproton $g$-factor.

Here, we review the first parts-per-billion measurement of the antiproton magnetic moment [14], which was performed in the BASE multi Penning-trap system [22]. The measurement is based on the observation of individual antiproton spin transitions [23] combined with a novel two-particle measurement technique. In addition, we compare our result to measurements of the proton magnetic moment $[10,11]$ and derive limits on CPT-odd interactions. We conclude the manuscript by discussing perspectives to further improve upon measurement precision.

\section{Methods}

The ideal Penning trap consists of a constant homogeneous magnetic field $B_{0}$ in the axial $z$-direction superimposed by an electrostatic quadrupole potential. The motion of single trapped particles in such a field configuration is a superposition of three independent eigenmotions [18]: the axial mode along the magnetic field lines at frequency $v_{z}$, and two radial modes, the modified cyclotron mode and the magnetron mode at frequencies $v_{+}$and $v_{-}$, respectively. The cyclotron frequency $v_{c}$ is related to the three eigenmotions by an invariance theorem [18]:

$$
v_{c}^{2}=v_{+}^{2}+v_{z}^{2}+v_{-}^{2} .
$$

Our cyclotron frequency measurements are based on the non-destructive detection of image currents using highly-sensitive superconducting detectors [24]. These enable us to determine $v_{z} \approx 675 \mathrm{kHz}$ in about $48 \mathrm{~s}$ with a resolution better than $30 \mathrm{mHz}$. The radial frequencies $v_{+}$and $v_{-}$are measured by coupling the radial and the axial modes with quadrupolar radiofrequency drives at $v_{+}-v_{z}$ and $v_{z}+v_{-}$, respectively. Alternating measurements of $v_{+}$and $v_{z}$ combined with occasional measurements of $v_{-}$allow the determination of $v_{c} \approx 29.7 \mathrm{MHz}$ with an uncertainty as low as $70 \mathrm{mHz}$ in about two minutes [25]. 
To measure the Larmor frequency $v_{L}$, we determine the spin-transition probability $P_{\mathrm{SF}}$ as function of a magnetic rf-drive at frequency $v_{r f}$. For this purpose, it is essential to nondestructively determine the antiproton's spin state. To this end, the continuous Stern-Gerlach effect [26] is applied, which uses a Penning trap with an intentionally superimposed inhomogeneous magnetic field $B_{z}=B_{0}+B_{2}\left(z^{2}-\rho^{2} / 2\right)$. This "magnetic bottle" couples the spin magnetic moment to the particle's axial oscillation frequency. Consequently, spin transitions are detected by observing a change of the axial frequency:

$$
\Delta v_{z, \mathrm{SF}}= \pm \frac{1}{2 \pi^{2}} \frac{\mu_{\bar{p}}}{m_{\bar{p}}} \frac{B_{2}}{v_{z}} .
$$

This principle has been successfully applied in measurements of the electron/positron magnetic moment. For protons and antiprotons, the ratio $\mu_{\bar{p}} / m_{\bar{p}}$ is however $\approx 10^{6}$ times smaller compared to the leptons and spin transitions are considerably harder to detect. Therefore, we use and ultra-strong magnetic inhomogeneity $B_{2}=272(12) \mathrm{kT} / \mathrm{m}^{2}$ to obtain a detectable frequency shift of $\Delta v_{z, \mathrm{SF}}=172(8) \mathrm{mHz}$. Such an "analysis trap" has been used for the first detection of spin transitions of a single proton using a statistical detection method [27]. We later applied this method to the antiproton and reported on a measurement of $\mu_{\overline{\mathrm{p}}}$ at a fractional precision of 0.8 parts in a million [13].

To significantly increase the measurement precision, it is essential to use multi-trap methods $[12,14,28,29]$. Here, the analysis trap serves merely as a spin-state analyser, whereas the precision frequency-ratio measurement $v_{L} / v_{c}$ is performed in a "precision trap", which has a $10^{5}$-fold more homogeneous magnetic field than the magnetic bottle trap. In short, a multi-trap measurement sequence starts with the particle's spin state identification in the analysis trap. In the next step the particle is transported to the precision trap where spin transitions are induced while the cyclotron frequency is measured. Afterwards, the particle is shuttled back to the analysis trap and the spin state is analyzed. Compared to direct measurements in the magnetic bottle, this approach dramatically reduces the linewidth of the $g$-factor resonance, enabling measurements at the parts-per-billion level. To apply such multi-trap methods, it is essential to stabilize the axial frequency fluctuation $\Xi_{z}$ in the analysis trap to $\Xi_{z} \ll \Delta v_{z}$,SF, being a crucial requirement to unambiguously determine the final spin state after the experimental procedures in the precision trap. This constitutes a major challenge and required development of several cutting-edge methods which are described in a sequence of publications $[23,27,29,30]$. By applying extensive sub-thermal cooling methods, we prepare a single antiproton with $E_{+} / k_{B}<100 \mathrm{mK}$. With particles at such low cyclotron energy, the axial frequency stability to obtain spin-state detection fidelities $>80 \%$ can be reached, which is sufficient to perform efficient multi-trap measurements.

\section{Experiment}

The relevant part of our Penning-trap system, which we used to apply the multi-trap method is shown in Fig. 1. We used three traps and two antiprotons in our measurement scheme: a hot "cyclotron antiproton" with $T_{+}=356(27) \mathrm{K}$ probes the magnetic field, whereas a cold "Larmor antiproton" with $E_{+} / k_{B}<100 \mathrm{mK}$ is used for the high-fidelity spin-transition spectroscopy. The first trap is the analysis trap to identify individual spin transitions of the Larmor antiproton. The second trap is the homogeneous precision trap used for the determination of the $g$-factor. It has a magnetic field of $B_{0}=1.945 \mathrm{~T}$ and a residual inhomogeneity of $B_{2}=2.7(3) \mathrm{T} / \mathrm{m}^{2}$. Both traps are equipped with an axial detection system at $675 \mathrm{kHz}$ 


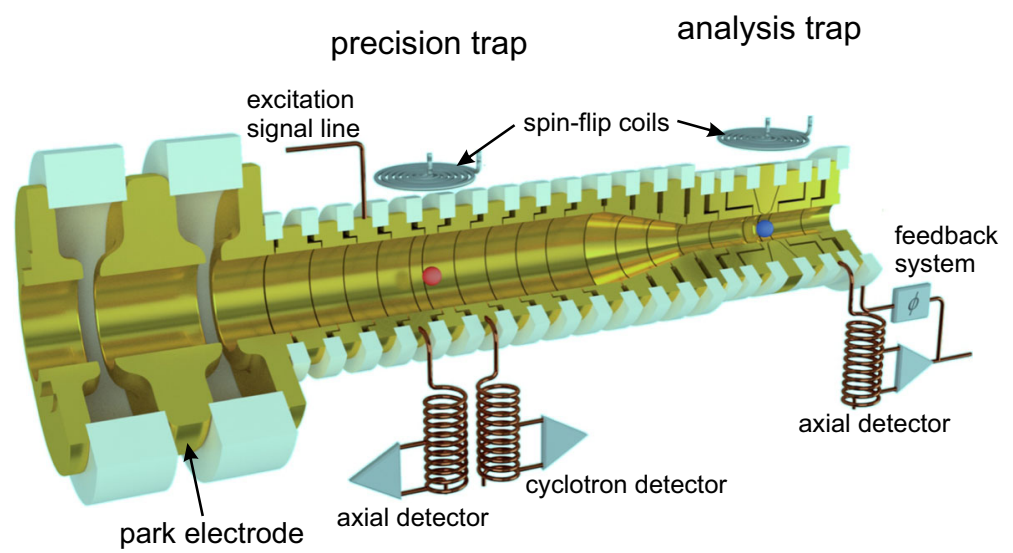

Fig. 1 Relevant part of the BASE Penning-trap system for the antiproton magnetic moment measurement showing the cyclotron antiproton (red) and the Larmor antiproton (blue) in the initial configuration of the measurement sequence

resonance frequency for precision frequency measurements. The precision trap has in addition a superconducting cyclotron detection system at $v_{+}=29.7 \mathrm{MHz}$, which is used for the preparation of the cold Larmor antiproton. The third trap in use is a park trap, which temporarily stores the cyclotron antiproton while the Larmor antiproton is in the precision trap.

The initial configuration of a measurement sequence is prepared by extracting a single antiproton as Larmor antiproton from the reservoir trap [31], resistively cooling it in the precision trap, and shuttling it to the analysis trap, until the antiproton is observed in a state with $E_{+} / k_{B}<100 \mathrm{mK}$ and $E_{-} / k_{B}<10 \mathrm{mK}$ [29]. Subsequently, the cyclotron antiproton is extracted from the reservoir and the radial modes are sideband cooled in the precision trap.

The detailed measurement sequence is illustrated in Fig. 2. It starts by initializing the spin state of the Larmor antiproton in the analysis trap. To this end, a sequence of axial frequency measurements is recorded. After every second frequency measurement, we irradiate a resonant spin-flip drive which saturates the Larmor resonance at a spin-flip probability $P_{\mathrm{SF}} \approx 0.5$. In this way, we simultaneously characterize the axial frequency fluctuation $\Xi_{z}$ and observe spin transitions. We repeat this sequence until a frequency shift $\left|\Delta v_{z}\right|>190 \mathrm{mHz}$ is observed. This initializes the spin-state to better than $98 \%$ fidelity. Examples of spin-state identification sequences are shown in Fig. 3. Subsequently, the spinflip attempt in the precision trap is started by measuring the magnetic field using a sequence of three cyclotron frequency measurements with the cyclotron antiproton. Next, the Larmor particle is transported to the precision trap while the cyclotron antiproton is moved into the park trap. A spin-flip drive at frequency $v_{r f}$ and a duration $t_{r f}=8 \mathrm{~s}$ is applied to probe the $g$-factor of the Larmor antiproton in the precision trap. The drive amplitude is chosen to slightly saturate the resonance to provide the maximum contrast of the resonance curve [14]. Afterwards, the two antiprotons are returned to their respective traps and the cyclotron frequency is measured in the precision trap three times with the cyclotron antiproton. Lastly, the final spin state of the spin-flip attempt in the precision trap is determined. In this case, the fidelity depends on occurrence of spin transitions during the first spin-flip drives and the random frequency fluctuations superimposed to the axial frequency jump induced by a spin-flip. Therefore, the fidelity is in general lower than for the first state initialization [23]. 


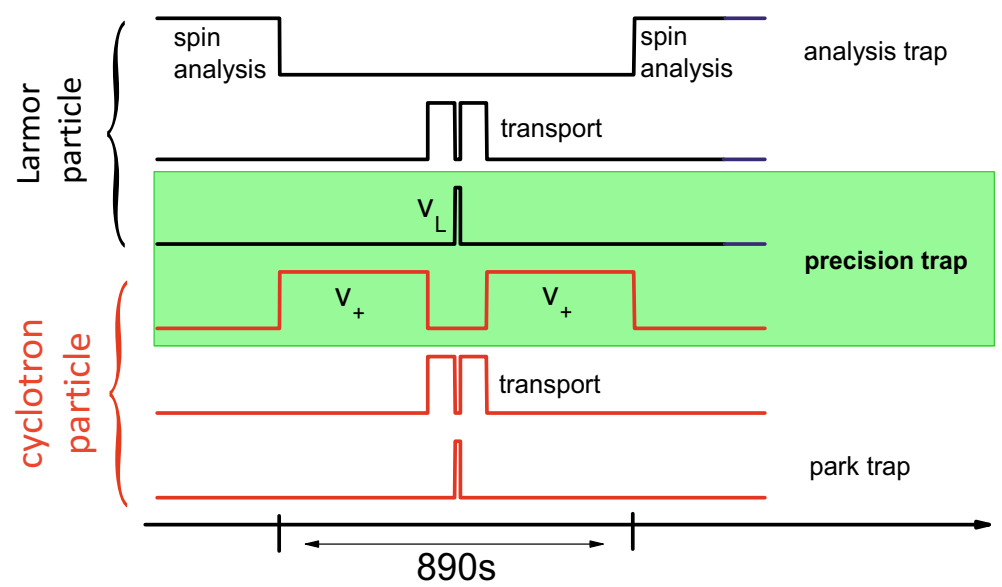

Fig. 2 Illustration of the measurement sequence of the antiproton magnetic moment measurement. The new method uses a cold "Larmor particle" for spin state detection and a hot "cyclotron particle" for magnetic field measurements. For further details see text

The shuttling to the precision trap and back causes fluctuations in the cyclotron energy $\Xi_{\Delta E_{+}} / k_{B}=22 \mathrm{mK}$. Eventually, the cyclotron energy can exceed the threshold of $200 \mathrm{mK}$ after several measurement cycles. This increases the axial frequency fluctuations and reduces the spin-state fidelity below $80 \%$. In this case, we interrupt the measurement sequence for re-cooling the cyclotron mode of the Larmor particle below the threshold. Compared to the established double Penning-trap method [28], however, the two particle technique allowed us to perform about 75 measurement cycles without cooling interruption. Overall, this greatly enhances the data accumulation rate of the experiment, and constitutes a crucial development towards the measurement reported here.

The objective of a measurement cycle is to determine the probability $P(\mathrm{SF})$ that the spinflip drive at the magnetic-field normalized frequency ratio $\Gamma=v_{r f} /\left\langle v_{c}\right\rangle$ in the precision trap caused a spin transition. $P(\mathrm{SF})$ is determined by the observed frequency shifts in the analysis trap. Two examples are shown in Fig. 3. Details on the calculation of $P(\mathrm{SF})$ are described in refs. $[14,23] .\left\langle v_{c}\right\rangle$ is the measured cyclotron frequency which is the average of all six cyclotron frequency measurements in a measurement cycle. Thereby, we compensate the linear magnetic field decay of 2.5(6) p.p.b./h of the superconducting magnet and reduce the contributions of cyclotron frequency measurement fluctuations to the linewidth of the $g$-factor resonance $\Xi_{B} / B=3.9(1)$ p.p.b.

The statistical evaluation of the antiproton $g$-factor is based on an un-binned likelihood analysis of 933 experiment cycles using a well understood line-shape function $P_{\mathrm{SF}}(\Gamma, g \bar{p})$ and the probabilities $P(\mathrm{SF})$ from the spin-state identification sequences. The line-shape $P_{\mathrm{SF}}(\Gamma, g \bar{p})$ is derived in ref. [32]. It is the convolution of the Rabi resonance with the Boltzmann distribution of the axial energy due to the interaction of the antiproton with the detection system. The residual magnetic field inhomogeneity combined with the thermal distribution of the axial energy causes decoherence and limits $P_{\mathrm{SF}} \leq 0.5$. In addition, we consider magnetic field fluctuations and sideband measurement fluctuations in the line-shape function [14]. From this evaluation, we obtain an antiproton $g$-factor of $2.7928473453(30)$. Considering the different orbits of the two antiprotons, our result 
(a)

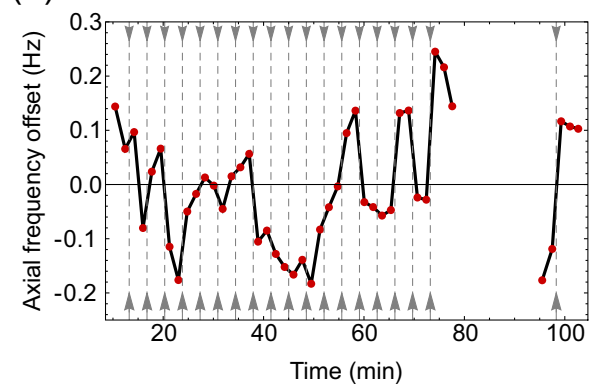

(b)

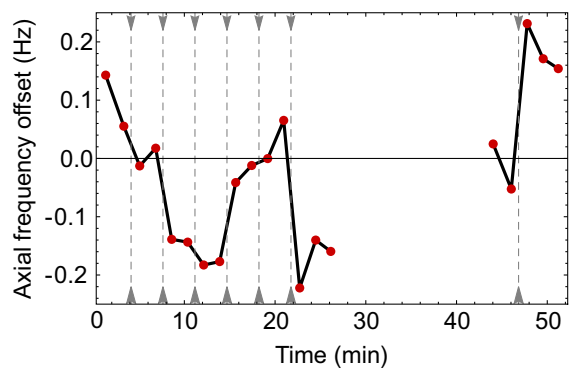

Fig. 3 Spin-state identification sequences to determine the probability that a spin flip occurred in the precision trap $P(\mathrm{SF})$ for two measurement sequences of the magnetic moment measurement. The red points show the measured axial frequencies, and the grey lines indicate when a resonant spin-flip drive with a spin-flip probability $P_{\mathrm{SF}} \approx 0.5$ was applied in the analysis trap. The axial frequency measurements are interrupted for the precision trap spin-flip attempt. In (a), spin transition occurred with a high probability $P(\mathrm{SF})=0.9995$, whereas in (b) the probability $P(\mathrm{SF})=6 \cdot 10^{-5}$ is very low

requires a systematic correction of $\delta g / g=-0.4(1.0)$ p.p.b., as discussed in detail in [14]. The final result is:

$$
g_{\bar{p}}=2.7928473441(42)
$$

with $68 \%$ C.L. Comparing the antiproton magnetic moment to the recent proton $g$-factor measurements $[10,11]$, the $g$-factor difference $\Delta g=\left(g_{p}-g \bar{p}\right)$ is less than one standard deviation.

To set constraints on CPT-odd interactions we use the $95 \%$ confidence limit on the $g$-factor difference

$$
\Delta g=\left(g_{\bar{p}}-g_{p}\right)=-0.5(7.3) 10^{-9},
$$

which represents the limit on a potential intrinsic difference of the proton and antiproton magnetic moments and on CPT-odd interactions. In a more microscopic picture, CPT-odd interactions would manifest themselves in a perturbation of the quantum-level structure of the trapped antiproton, compare Fig. 4. Our measurement primarily tests whether the spinlevel spacing for protons and antiprotons is modified by any CPT-odd interaction $V_{\mathrm{CPT} \text {,odd }}$. Corresponding limits are usually expressed in units of frequency or energy resolution:

$$
\begin{aligned}
\Delta v_{L, \bar{p}} & =\sigma\left(g_{\bar{p}}\right) v_{c}=215 \mathrm{mHz}, \\
\hbar \Delta \omega_{L, \bar{p}} & =9 \cdot 10^{-25} \mathrm{GeV} .
\end{aligned}
$$

Different potentially existing CPT-odd extensions $V_{\mathrm{CPT} \text {,odd }}$ to the Standard Model have been described [19-21]. In the minimal Standard Model Extension [20], the Larmor frequency of the proton/antiproton is modified by $\Delta v_{L}=4 b_{3}$, where $b_{3}$ is the component of the Lorentzand CPT-violating vector $\mathbf{b}$ parallel to the magnetic field. Depending on the orientation of $\mathbf{b}$ relative to the experiment, stationary magnetic moment differences and/or oscillations of the level spacing with periods related to the earth's interstellar orbit are expected. Recently, the non-minimal Standard Model extension has been developed, which includes also higher dimensional operators and separate coefficients for particle and antiparticle tests and extends the spectrum of possible CPT violating physics [21]. Using the measured uncertainty of $\Delta g$, we derive limits on non-minimal SME coefficients, e.g. for $\left|\tilde{b}_{p}^{Z *}\right|$ :

$$
\left|\tilde{b}_{p}^{Z *}\right|<1.4 \cdot 10^{-24} \mathrm{GeV},
$$

limits to other coefficients are summarized in [14]. 


\section{proton}

spin

\section{cyclotron}

\section{antiproton}

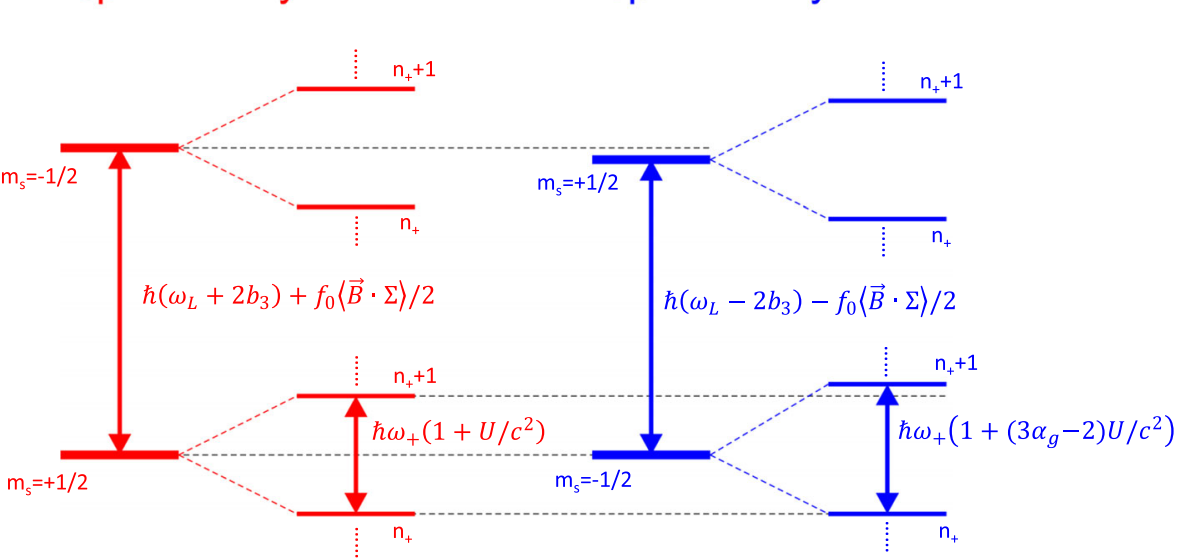

Fig. 4 Relevant part of the quantum level structure of a trapped proton and antiproton in a Penning trap. Here, we show the modifications expected from the effects discussed in refs. [19, 20]. A discussion of the gravitational redshift modifying the cyclotron ladder spacing is found in ref. [25]

A different approach on CPT violation is discussed in ref. [19], where the impact of CPTodd dimension-five operators on magnetic moment measurements is investigated. One of these operators $V_{C P T, \text { odd }}=f_{0, p}(\vec{\sigma} \cdot \vec{B})$ causes a stationary splitting in particle/antiparticle $g$-factors. Here, $\vec{\sigma}$ and $\vec{B}$ are the spin and magnetic field vectors, respectively. The coupling strength $f_{0, p}$ is constrained by our measurements to:

$$
f_{0, p}<2.4 \cdot 10^{-12} \mu_{B},
$$

where $\mu_{B}$ is the Bohr magneton. The energy resolution of the measurement reported here reaches comparable limits as derived from $\mu^{+} / \mu^{-}\left(f_{0, \mu}<8 \cdot 10^{-11} \mu_{B}\right)$ [33] and $e^{+} / e^{-}$ $\left(f_{0, e}<2.3 \cdot 10^{-12} \mu_{B}\right)$ [34] magnetic moment measurements. In summary, we have improved our limits on CPT-odd interactions in the baryon sector by a factor 350 based on our recent measurements of the proton and antiproton magnetic moments [11, 14], and by a factor of about 3000 compared to the best competing measurement [17].

\section{Perspectives}

Future perspectives of our experiment target the improvement of the relative precision in our experiments. We are planning to implement phase methods developed in $g$-factor measurements of electrons in highly-charged ions, which reached a relative precision of $2 \cdot 10^{-11}[35$, 36]. In addition, we are currently developing sympathetic cooling techniques for protons and antiprotons by means of laser cooled beryllium ions [37, 38]. These developments will improve the spin-state fidelity and the measurement precision, and eliminate at least $50 \%$ of the time budget of the recent $g$-factor measurements. Ultimately, the measurements using our antiproton apparatus in the antiproton decelerator environment will be limited by the external magnetic field fluctuations of $\sigma_{B} / B=3.9(1)$ p.p.b. in the AD hall. Better suppression of these fluctuations is certainly possible by improving the active and passive magnetic 


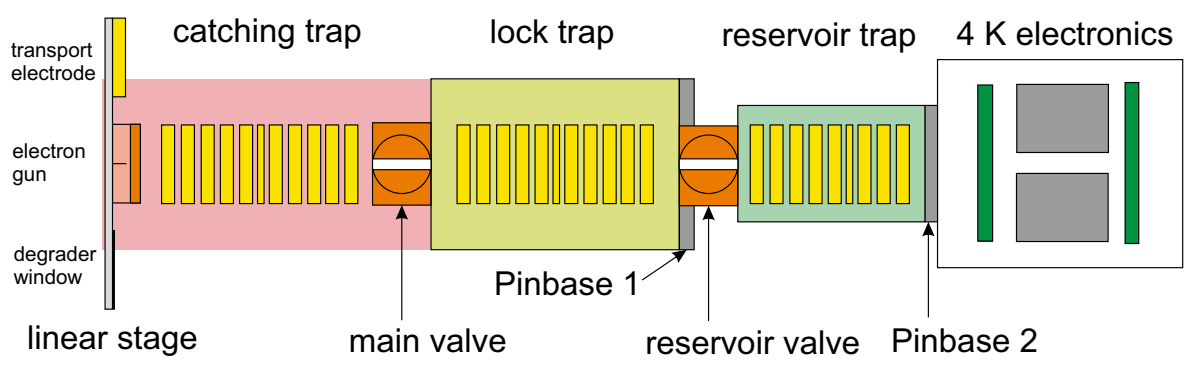

$\begin{array}{llll}\text { isolation vacuum } & \text { vacuum lock } & \text { ultra-low vacuum } & \text { image current } \\ \text { catching } & \text { antiproton counting } & \text { cooling/storage } & \text { detectors } \\ \text { vacuum monitoring } & \text { vacuum monitoring } & \text { separation } & \text { amplifiers/filters }\end{array}$

Fig. 5 Schematic of the Penning-trap system for the antiproton transport container

shielding. On the long-term, we plan to relocate antiprotons to dedicated precision laboratories and to conduct antiproton precision measurements in a calm magnetic environment. Therefore, we target the development of a transportable antiproton container, see Fig. 5, which can be loaded at the AD/ELENA facility and subsequently relocated to transfer the antiprotons into a precision experiment. BASE has developed all required methods to realize such a transportable device - including methods to supply an antiproton precision experiment for more than a year [15], non-destructive single antiproton extraction from a reservoir [31], as well as adiabatic particle shuttling [12]. The successful implementation of such a device will enable us to supply the BASE experiments at Mainz and Hannover with a local source of antiprotons, which would considerably extend the BASE experimental program.

In conclusion, we have discussed limits on CPT-odd interactions set by our recent 1.5 p.p.b. antiproton magnetic moment measurement. We target the development of several new methods to further extend the sensitivity of testing fundamental symmetries with antiprotons potentially by another factor 10 to 100 in the next years.

Acknowledgments We acknowledge technical support from the Antiproton Decelerator group, CERN's cryolab team, and all other CERN groups which provide support to Antiproton Decelerator experiments. We acknowledge financial support from the RIKEN Initiative Research Unit Program, RIKEN President Funding, RIKEN Pioneering Project Funding, RIKEN FPR Funding, RIKEN chief scientist program, the RIKEN JRA Program, the Max-Planck Society, the EU (ERC Advanced Grant No. 290870-MEFUCO), and the Helmholtz-Gemeinschaft.

Open Access This article is distributed under the terms of the Creative Commons Attribution 4.0 International License (http://creativecommons.org/licenses/by/4.0/), which permits unrestricted use, distribution, and reproduction in any medium, provided you give appropriate credit to the original author(s) and the source, provide a link to the Creative Commons license, and indicate if changes were made.

\section{References}

1. Hori, M., Walz, J.: Prog. Part. Nucl. Phys. 72, 206-253 (2013)

2. Hori, M., et al: Science 354, 610-614 (2016)

3. Ahmadi, M., et al: Nature 529, 373-376 (2016)

4. Ahmadi M., et al: Nature. https://doi.org/10.1038/s41586-018-0017-2 (2018)

5. Ahmadi, M., et al: Nature 483, 439-443 (2012) 
6. Malbrunot, C., et al: Phil. Trans. R. Soc. A 376, 20170273 (2017)

7. Kellerbauer, A., et al.: Nucl. Instr. Meth. B 266, 351-356 (2008)

8. Perez, P., et al: Nucl. Instr. Meth. A 545, 20-30 (2005)

9. Bertsche, W.A., et al: Phil. Trans. R. Soc. A 376, 20170265 (2018)

10. Mooser, A., et al: Nature 509, 596-599 (2014)

11. Schneider, G., et al: Science 358, 1081-1084 (2017)

12. Ulmer, S., et al: Nature 524, 196-199 (2015)

13. Nagahama, H., et al: Nat. Commun. 8, 14084 (2017)

14. Smorra, C., et al: Nature 350, 371-374 (2017)

15. Sellner, S., et al: New J. Phys. 19, 083023 (2017)

16. Gabrielse, G., et al: Phys. Rev. Lett. 82, 3198 (1999)

17. DiSciacca, J., et al: Phys. Rev. Lett. 110, 130801 (2013)

18. Brown, L.S., Gabrielse, G.: Rev. Mod. Phys. 58, 233 (1986)

19. Stadnik, Y.V., et al: Phys. Rev. D 90, 045035 (2014)

20. Bluhm, R., et al: Phys. Rev. D 57, 3932-3943 (1998)

21. Ding, Y., Kostelecky, V.A.: Phys. Rev. D 94, 056008 (2016)

22. Smorra, C., et al: Eur. Phys. J. Special Topics 224, 3055-3108 (2015)

23. Smorra, C., et al: Phys. Lett. B 769, 1-6 (2017)

24. Nagahama, H., et al: Rev. Sci. Instr. 87, 113305 (2016)

25. Higuchi, T., et al: LEAP proceedings, This issue (2018)

26. Dehmelt, H., Ekström, P.: Bull. Am. Phys. Soc. 18, 72 (1973)

27. Ulmer, S., et al: Phys. Rev. Lett. 106, 253001 (2011)

28. Häffner, H., et al: Eur. Phys. J. D - At. Mol. Opt. Plas. Phys. 22, 163 (2003)

29. Mooser, A., et al: Phys. Lett. B 723, 78-81 (2013)

30. Mooser, A., et al: Phys. Rev. Lett 110, 140405 (2013)

31. Smorra, C., et al: Int. J. Mass Spectr. 389, 10-13 (2015)

32. Brown, L.S.: Ann. Phys. 159, 62 (1985)

33. Bennett, G.W., et al: Phys. Rev. D 73, 072003 (2006)

34. Mittleman, R., et al: Phys. Rev. Lett. 83, 2116 (1999)

35. Sturm, S., et al: Phys. Rev. A 87, 030501 (2013)

36. Heie, F., et al: Phys. Rev. Lett. 119, 033001 (2017)

37. Bohman, M., et al: J. Mod. Opt. 65, 568-576 (2018)

38. Niemann, M., et al.: https://doi.org/10.1142/9789814566438_0011 (2013) 